\title{
The Fulde-Ferrell-Larkin-Ovchinnikov Superconductivity in Disordered Systems
}

\author{
A. PтOK* \\ Institute of Physics, University of Silesia, Uniwersytecka 4, 40-007 Katowice, Poland
}

\begin{abstract}
The Fulde-Ferrell-Larkin-Ovchinnikov phase, with a spatially oscillating order parameter, may be induced by strongly magnetic field at low temperature. It is believed that the Fulde-Ferrell-Larkin-Ovchinnikov phase can exist only in homogeneous superconductors, and even weak impurity potential can lead to its destruction. The analysis of the Fulde-Ferrell-Larkin-Ovchinnikov phase in the Bogoliubov-de Gennes equation shows however, that this phase can exist in the presence of weak disorder. Using Bogoliubov-de Gennes equations, we discussed the influence of diagonal and off-diagonal disorder on the Fulde-Ferrell-Larkin-Ovchinnikov phase.
\end{abstract}

PACS numbers: 74.20.Fg, 74.20.Mn, 74.20.Rp, 74.25.Dw, 74.81.-g

\section{Introduction}

In the 1960's Fulde and Ferrell [1] along with Larkin and Ovchinnikov [2] suggested the possibility of existence of an unconventional superconducting state with a spatially oscillating order parameter (OP). This phase, called the Fulde-Ferrell-Larkin-Ovchinnikov phase (FFLO), is characterized by changes of the sign of $\mathrm{OP}$ in real space. This phase occurs at low temperature in the presence of a strong magnetic field. In the FFLO phase, the Cooper pairs have non-zero center-of-mass momenta [3]. It is a widely held belief that the FFLO phase can exist only in homogeneous superconductors, and even weak inhomogeneity can lead to its destruction [4]. However, the analysis of the FFLO phase carried out within the method of the Bogoliubov-de Gennes (BdG) equations, has shown that this phase can also exist in the presence of weak disorder. It is equally possible for the $s$-wave $[6,5]$ and the $d$-wave superconductors [7-9].

In the FFLO phase the OP can be expressed as a composition of plane waves

$$
\Delta\left(\boldsymbol{R}_{j}\right) \equiv\left\langle c_{j \downarrow} c_{j \uparrow}\right\rangle=\sum_{m=1}^{M} \Delta_{\boldsymbol{Q}_{m}} \exp \left(\mathrm{i} \boldsymbol{Q}_{m} \cdot \boldsymbol{R}_{j}\right),
$$

where $\boldsymbol{Q}_{m}$ represent the considered values of the Cooper pairs momentum. Analysing the superconducting phase, we will discuss: the BCS phase (for $M=1$ and $\boldsymbol{Q}_{1}=\mathbf{0}$, resulting in constant value of the $\mathrm{OP}$ ), the $\mathrm{FF}$ phase (for $M=1$ and $\boldsymbol{Q}_{1} \neq \mathbf{0}$, resulting in $\left.\Delta\left(\boldsymbol{R}_{j}\right) \sim \exp \left(\mathrm{i} \boldsymbol{Q}_{1} \cdot \boldsymbol{R}_{j}\right)\right)$, the LO phase (for $M=2$ and $\boldsymbol{Q}_{1}=-\boldsymbol{Q}_{2} \neq \mathbf{0}$, resulting in $\left.\Delta\left(\boldsymbol{R}_{j}\right) \sim \cos \left(\mathrm{i} \boldsymbol{Q}_{1} \cdot \boldsymbol{R}_{j}\right)\right)$ and the FFLO phase in general (for unconstrained real space profile of $\Delta\left(\boldsymbol{R}_{j}\right)$ ). The major question is what influence the chosen value of $M$

\footnotetext{
* e-mail: aptok@phys.us.edu.pl
}

has on the stability of the FFLO phase in inhomogeneous systems.

\section{Model}

We consider a model with local on-site pairing interaction, described by the Hamiltonian

$$
\begin{aligned}
H & =\sum_{\langle i, j\rangle, \sigma}\left(-t_{i j}\right) c_{i \sigma}^{\dagger} c_{j \sigma}+\sum_{i, \sigma}\left(-\mu-\sigma h+w_{i}\right) c_{i \sigma}^{\dagger} c_{i \sigma} \\
& +U \sum_{i} c_{i \uparrow}^{\dagger} c_{i \uparrow} c_{i \downarrow}^{\dagger} c_{i \downarrow}
\end{aligned}
$$

where $t_{i j}$ is the nearest-neighbor (NN) hopping integral between the $i$-th and $j$-th site, $\mu$ is the chemical potential, $h$ is the Zeeman term for external magnetic field, $w_{i}$ is the impurity potential at site $i$, and $U<0$ denotes pairing potential. Recent investigations of the FFLO phase in the attractive Hubbard model have been motivated mostly by the increasing interest in the ultracold Fermi gases $[10,11]$. These approaches may also be applicable to compounds other than the strongly correlated heavy fermion systems $[6,12]$, where intersite pairing should be considered.

In our approach we neglect electronic correlations, which in the presence of magnetic field may lead to spin-dependent effective mass [13]. Due to this effect, FFLO sets on at much weaker magnetic field, when compared to the non-interacting case. Similar effect may originate also from the repulsive pair hopping interaction [14]. Moreover, electronic correlations may lead to a highly non-trivial interplay between magnetism and the FFLO superconductivity [15]. In disordered systems one may also expect that the pairing interaction becomes a site-dependent quantity [16]. An additional limitation of the present approach originates from the assumption that the magnetic field is parallel to the superconducting plane. 
Otherwise, orbital effects should be explicitly taken into account [17].

We examine two types of disorder: diagonal and off-diagonal ones. We assume that in an inhomogeneous system the sources of the disorder are nonmagnetic impurities located in random sites of the lattice. In the case of the diagonal disorder we assume that $t_{i j}=t_{0}$ for all sites. At sites where the impurities are located, we assume that $w_{i}=w \neq 0$. In other sites $w_{i}=0$. For the off-diagonal disorder we assume that $w_{i}=0$. We consider the hopping integral between the NN lattice sites. If impurities are absent at sites $i$ and $j$, then: $t_{i j}=t_{0}$. In other cases $t_{i j}=t_{0}+\Delta t$, where $\frac{\Delta t}{t_{0}} \in(0,-1)\left(t_{0}\right.$ is the energy unit).

The Hamiltonian given by Eq. (2) can be diagonalized in the mean-field approximation through the following transformation:

$$
c_{k \sigma}=\sum_{n}\left(u_{k n \sigma} \gamma_{n \sigma}-\sigma v_{k n \sigma}^{*} \gamma_{n \bar{\sigma}}^{\dagger}\right),
$$

where $\gamma_{n \sigma}$ and $\gamma_{n \sigma}^{\dagger}$ are the quasiparticle operators, $u_{k n \sigma}$ and $v_{k n \sigma}$ are the BdG eigenvectors. We then obtain the self-consistent BdG equations

$$
E_{n \sigma}\left(\begin{array}{c}
u_{i n \sigma} \\
v_{i n \bar{\sigma}}
\end{array}\right)=\sum_{j}\left(\begin{array}{cc}
H_{i j \sigma} & \Delta_{i j} \\
\Delta_{i j}^{*} & -H_{i j \bar{\sigma}}^{*}
\end{array}\right)\left(\begin{array}{c}
u_{j n \sigma} \\
v_{j n \bar{\sigma}}
\end{array}\right),
$$

where $H_{i j \sigma}=-t_{i j}+\left(-\mu-\sigma h+w_{i}\right) \delta_{i j}$ is the single particle Hamiltonian, $\Delta_{i j}=U \Delta_{\boldsymbol{R}_{j}} \delta_{i j}$ is the OP with $s$-wave symmetry. Using the BdG equations, we analyze spatial profile of the OP.

\section{Details of calculations}

We investigate two cases, in which the OP is given as in the FFLO phase and the LO phase. For a system with the FFLO phase, the spatial decomposition of the OP is unconstrained. Then, all values of the Cooper pair momenta are admitted

$$
\begin{aligned}
& \Delta\left(\boldsymbol{R}_{j}\right)=\left\langle c_{j \downarrow} c_{j \uparrow}\right\rangle \\
& \quad=\sum_{n}\left[u_{j n \uparrow} v_{j n \downarrow}^{*} f\left(E_{n \uparrow}\right)-u_{j n \downarrow} v_{j n \uparrow}^{*} f\left(-E_{n}\right)\right],
\end{aligned}
$$

where $f(E)=\frac{1}{\exp (\beta E)+1}$ is the Fermi-Dirac distribution function. For a system with the LO phase, we take two opposite values of Cooper pair momenta. For this sake we calculate the Fourier transform of the following quantity:

$$
\begin{aligned}
& \tilde{\Delta}\left(\boldsymbol{R}_{j}\right)=\sum_{n}\left[u_{j n \uparrow} v_{j n \downarrow}^{*} f\left(E_{n \downarrow}\right)-u_{j n \downarrow} v_{j n \uparrow}^{*} f\left(-E_{n}\right)\right] \\
& =\sum_{m=1}^{M} \Delta_{m} \exp \left(\mathrm{i} \boldsymbol{Q}_{m} \cdot \boldsymbol{R}_{j}\right) .
\end{aligned}
$$

We choose two values of $m, m_{1}$ and $m_{2}$, such that $\Delta_{m}$ is maximal and $\boldsymbol{Q}_{m_{1}}=-\boldsymbol{Q}_{m_{2}}=\boldsymbol{Q}$. Then, the OP takes on a standard form for the LO phase

$$
\Delta_{\boldsymbol{R}_{j}}=2 \Delta_{0} \cos \left(\mathrm{i} \boldsymbol{Q} \cdot \boldsymbol{R}_{j}\right) \text {. }
$$

Numerical calculations were performed for the 2D square lattice: $N_{x}=32, N_{y}=30$, with periodic boundaries. The impurities were located in 51 random lattice sites (which corresponds to a $5.5 \%$ concentration). Additionally we impose: $U=-2.0 t_{0}, \mu=-0.4 t_{0}$ and $k T=0$.

\section{Numerical results and discussion}

The comparison of the phase diagrams obtained for the LO and the FFLO phases (Fig. 1) shows a significant difference between critical magnetic fields. This difference originates from the restriction imposed on the allowed momenta of the Cooper pairs in the case of LO superconductivity. This, in turn, causes a large decrease of the critical magnetic field, both for diagonal and off-diagonal disorder. Higher critical magnetic field obtained for the FFLO phase originates from the fact that the impurities modify the spatial profile of the superconducting OP, as presented in Fig. 2 and Fig. 3. One can see that the resulting profile of the OP in the FFLO phase significantly differs from the cosine function that is characteristic for the LO phase. Then, the Fourier decomposition of $\Delta\left(\boldsymbol{R}_{j}\right)$ for the FFLO phase, given by Eq. (6), consists of many modes. The projection on the LO phase (see Eq. (7)) lowers the magnitude of the $\mathrm{OP}$, and, as a consequence, is responsible for lower stability of the LO superconductivity.

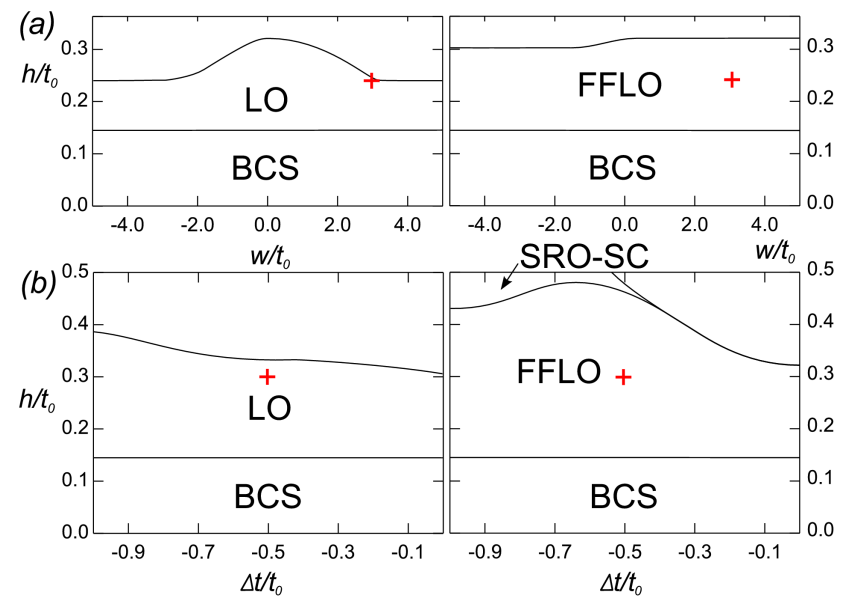

Fig. 1. Phase diagrams for diagonal (a) and off-diagonal (b) disorder. Left parts depict results for the LO phase, and right parts for the FFLO phase. SRO-SC stands for superconductivity with short range order, i.e., $\Delta\left(\boldsymbol{R}_{j}\right) \neq 0$ only in the vicinity of impurities. Crosses mark the parameters for the results presented in Fig. 2 and Fig. 3.

In the case of the diagonal disorder (Fig. 2b), one can observe a decrease of the OP in the vicinity of impurities. Eventually for strong impurity potential the OP vanishes at these sites. This result originates from modification of the local charge concentration. On the other hand, in the case of the off-diagonal disorder (Fig. 3b), the $\mathrm{OP}$ increases in the vicinity of impurities. For strong magnetic field $\Delta\left(\boldsymbol{R}_{j}\right)$ is non-zero only at site located close to impurities (SRO-SC phase in Fig. 1b). It appears as a result of relative increase of the pairing potential 


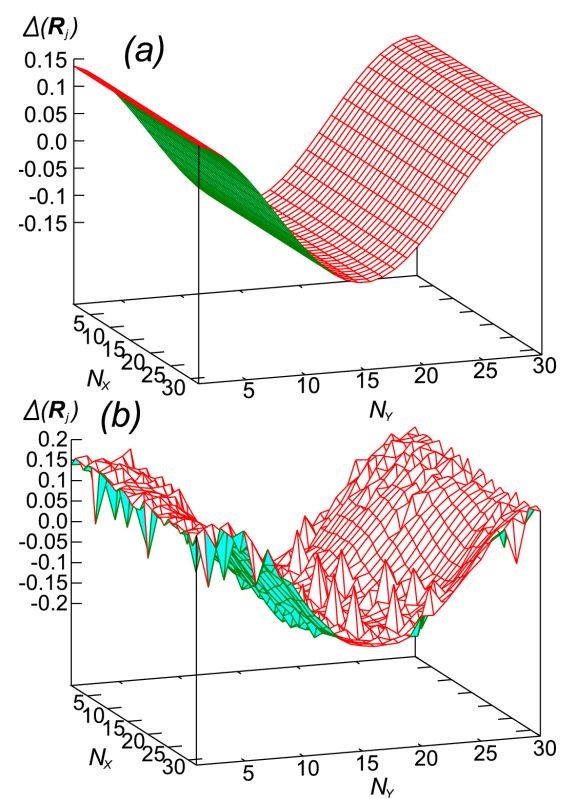

Fig. 2. Spatial profile of the OP in the case of diagonal disorder for $h=0.24 t_{0}$ and $w=3 t_{0}$. Part (a) depicts results for the LO phase, and part (b) for the unrestrained FFLO phase.

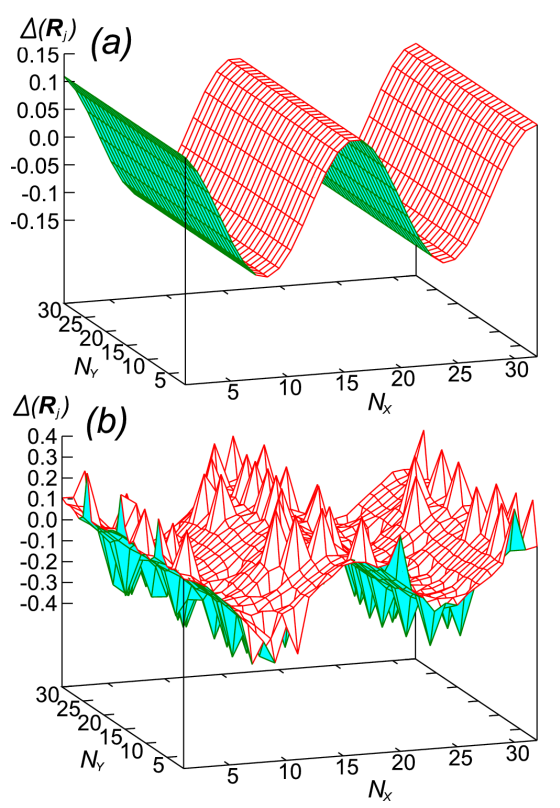

Fig. 3. Spatial profile of the OP in the case of off-diagonal disorder for $h=0.3 t_{0}$ and $\Delta t=-0.5 t_{0}$. Part (a) depicts results for the LO phase, and part (b) for the unrestrained FFLO phase. relative to a modified hopping integral between the site with impurity and its NN.

In this paper we used the BdG equations to discuss the influence of the diagonal and the off-diagonal disorder on the FFLO phase. We have demonstrated that, in the presence of impurities, the standard LO phase may be unstable against formation of the FFLO superconductivity with a much more complicated spatial profile of the OP. Hence, when analysing the FFLO phase in inhomogeneous systems, we must consider all possible Cooper pair momenta in the Fourier decomposition of the OP (all possible values of $M$ in Eq. (1)).

\section{References}

[1] P. Fulde, R.A. Ferrell, Phys. Rev. 135, A550 (1964).

[2] A.I. Larkin, Yu.N. Ovchinnikov, Zh. Eksp. Teor. Fiz. 47, 1136 (1964) [Sov. Phys. JETP 20, 762 (1965)].

[3] Y. Matsuda, H. Shimahara, J. Phys. Soc. Jpn. 76, 051005 (2007) and references therein.

[4] L.G. Aslamazov, Zh. Eksp. Teor. Fiz. 55, 1477 (1968) [Sov. Phys. JETP 28, 773 (1969)].

[5] P. Dey, S. Basu, R. Kishore, J. Phys., Condens. Matter 21, 355602 (2009).

[6] Q. Cui, K. Yang, Phys. Rev. B 78, 054501 (2008).

[7] Q. Wang, C.-R. Hu, C.-S. Ting, Phys. Rev. B 75, 184515 (2007).

[8] Y. Yanase, New J. Phys. 11, 055056 (2009).

[9] Q. Wang, C.-R. Hu, C.-S. Ting, Phys. Rev. B 74, 212501 (2006).

[10] M. Rizzi, M. Polini, M.A. Cazalilla, M.R. Bakhtiari, M.P. Tosi, R. Fazio, Phys. Rev. B 77, 245105 (2008).

[11] T.K. Koponen, T. Paananen, J.-P. Martikainen, M.R. Bakhtiari, P. Törmä, New J. Phys. 10, 045014 (2008).

[12] Q. Wang, H.-Y. Chen, C.-R. Hu, C.S. Ting, Phys. Rev. Lett. 96, 117006 (2006).

[13] J. Kaczmarczyk, J. Spałek, Phys. Rev. B 79, 214519 (2009).

[14] A. Ptok, M.M. Maśka, M. Mierzejewski, J. Phys., Condens. Matter 21, 295601 (2009).

[15] M. Mierzejewski, A. Ptok, M.M. Maśka, Phys. Rev. B 80, 174525 (2009).

[16] M.M. Maśka, Ż. Śledź, K. Czajka, M. Mierzejewski, Phys. Rev. Lett. 99, 147006 (2007).

[17] M.M. Maśka, M. Mierzejewski, Phys. Rev. B 64, 064501 (2001). 